\title{
M2M Networking Architecture for Data Transmission and Routing
}

\author{
Soujanya Ambala \\ M.Tech (Software Engineering), \\ Assistant Professor, Siddhartha \\ Institute of Technology \& \\ Sciences, Hyderabad, \\ Telangana, India
}

\author{
Dr. Srinivas Ambala \\ M.Tech (CSE), Associate \\ Professor, Vignan's Institute of \\ Management and Technology for \\ Women, Ghatkesar, Telangana, \\ India
}

\author{
Sreedhar Ambala \\ M.Tech (CSE), Assistant Professor \\ Siddhartha Institute of Technology \\ \& Sciences, Hyderabad, \\ Telangana, India
}

\begin{abstract}
We propose a percolation based $\mathrm{M} 2 \mathrm{M}$ networking architecture and its data transmission method. The proposed network architecture can be server-free and router-free, which allows us to operate routing efficiently with percolations based on six degrees of separation theory in small world network modeling. The data transmission can be divided into two phases: routing and data transmission phase. In the routing phase, probe packets will be transmitted and forwarded in the network thus path selections are performed based on small-world strategy. In the second phase, the information will be encoded, say, with the fountain codes, and transmitted using the paths selected at the first phase. In such a way, an efficient routing and data transmission mechanism can be built, allowing us to construct a low-cost, flexible and ubiquitous network. Such a networking architecture and data transmission can be used in many M2M communications, such as the stub network of internet of things, and deep space networking, and so on.
\end{abstract}

Keywords: Machine-to-machine, percolation networking, fountain codes, internet of things.

\section{Introduction}

The stub network of internet of things (IoT) may have a lot of sub-netwoks buliding with machine-tomachine (M2M) communication systems, which are usually highly dynamic. That is, networking nodes and links may frequently join to the network and exit from the network. In addition, such networks are often composed using relatively inexpensive nodes that have low power consupmtion, low processing power, and bandwidth. The conventional internet architecture and TCP/IP are not suitable for the the applications of M2M networks because such conventional networks require a lot of servers and routers, and the high overhead in their protocols result in high bandwidth needs which is not reasonable for bandwidth limited communications. Therefore, it is necessary to examine the efficient network architecture and effective data transmission method to achieve better performance with low cost for the M2M communications.

\section{Proposed Machine-to-machine Network Architecture Based on "6-degrees" of Distance Rule}

A typical M2M network system with small-world properties of M2M network is usually composed of hundreds to thousands of, or even more nodes, and each node can router-freely communicate with several other neighbor nodes. Each node in the network connected to several neighbor nodes randomly. In such a way, a small-world network is formed, in which efficient routing can be operated with percolations based on the six degrees of separation [12].

\section{M2M Network Topology Based on "6-degrees" of Distance Rule}

Due to the low-power of M2M devices and the signalattenuation nature of wireless signals, a M2M node can directly communicate with some nodes nested within networks of neighborhood relations, while it is hard to communicate with the other nodes that are out 
the scope of radio range [13]. Therefore we take the network structure shown in Fig. 1 as an example of the M2M network, whose nodes live in a small world where the members are not tightly connected in some part. Each node may have direct connections with one or several other neighbor nodes which we refer this as "close-neighbors". For example, the nodes T, U, O, K and $\mathrm{M}$ live in neighborhoods and they are closeneighbors of the node $\mathrm{L}$, which means $\mathrm{T}, \mathrm{U}, \mathrm{O}, \mathrm{K}$ and $\mathrm{M}$ can send data packets to $\mathrm{L}$ directly.

Such a network should have the property of social network properties, in which the relations between $\mathrm{R}$ and $\mathrm{Q}$ (also between $\mathrm{P}$ and $\mathrm{J}$, and between $\mathrm{I}$ and $\mathrm{H}$ ) may represent the leisure relations. Moreover, T, S, O, $\mathrm{K}, \mathrm{L}, \mathrm{M}, \mathrm{N}$, and $\mathrm{U}$ may represent the close relations of a family. In such networks, one node may form ties with leisure or close relations with neighbor nodes nested within them. When the number of closeneighbors of one node is larger than the average close-neighbor number of all the network nodes, we define the node as "Star node", like node G in Fig. 1. When one node is not a close-neighbor of another node and if the two nodes want to communicate with each other, they can communicate with each other through relay nodes. Note that, when E wants to communicate with the node $\mathrm{N}$, it may 137 first "guess" that the node L is a close-neighbor of the node E, so it hands its data to the node L. Similarly, the node L "guesses" that the node $\mathrm{T}$ may directly connects to the node E, so it forwards the data to the node $\mathrm{T}$. Finally, the node $\mathrm{N}$ delivers the data packets to the node E, and vice versa. The one-way or twoway communications are then built between the source and destination nodes based on percolation method and the six degrees of separation principle, and any node can reach everywhere in finite steps of relaying with a very high probability [6][12][13]. In the network, each node acts as relay for the other nodes, it simultaneously transmits its own data packets, thus no dedicated routers are needed. As stated, the routing may be based on "guess", just like the greedy forwarding algorithm which selects the nearest node to the destination node as a relay node within its transmission range, following the rule of the best likelihood [14][15].

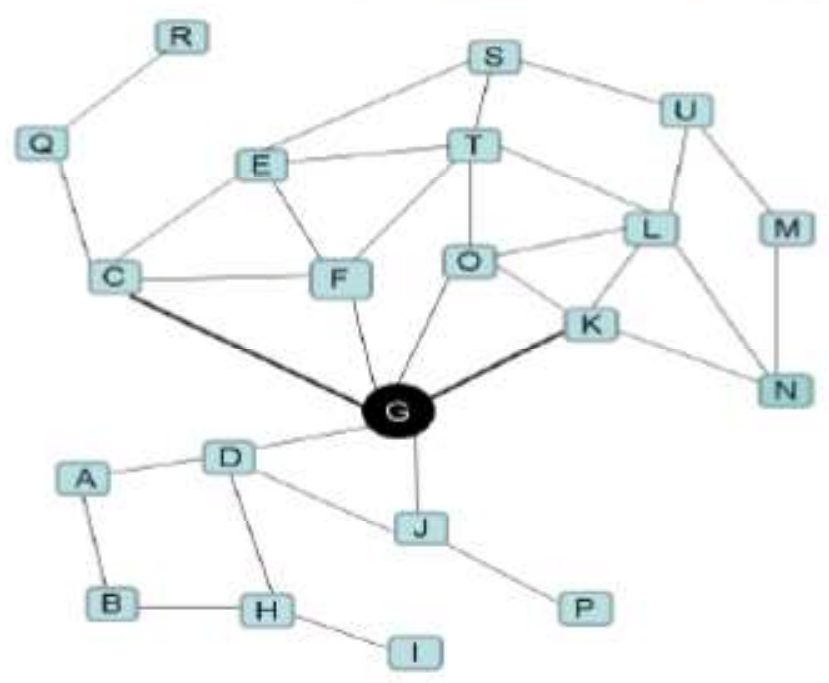

Fig. 1. An example of M2M network architecture with small-world properties

\subsection{Routing Information Table of M2M network}

In Fig. 1, each node will hand its data to its closeneighbors which can forward the data to the destination with high probability. Each node may maintain and store a routing information table which contains all or part of the following messages is listed

Table 1.

Table 1. Routing information table of network nodes of a "small-world" M2M network topology

\begin{tabular}{|l|l|l|}
\hline Field name & Symbol & Descriptions \\
\hline $\begin{array}{l}\text { Numes of } \\
\text { close-neighbor } \\
\text { nodes }\end{array}$ & $L_{1} L_{2} \ldots L_{N}$ & Name the close-eighbor nodes of the source node as $L$ \\
\hline $\begin{array}{l}\text { Number of } \\
\text { close-neighbor } \\
\text { nodes }\end{array}$ & $N$ & $\begin{array}{l}\text { This field describes the number of nodes available for direct } \\
\text { communication without relay. }\end{array}$ \\
\hline $\begin{array}{l}\text { Addresses of } \\
\text { close-neighbor }\end{array}$ & $A_{j} A_{2} \ldots A_{N}$ & $\begin{array}{l}\text { An address of a node is the identity number of a node. } \\
\text { Generally, the name and the address can be the same thus the }\end{array}$ \\
\hline
\end{tabular}




\subsection{Network Initialization Process of M2M network}

The network initialization process is as follows.

A node sends the query signal to its close neighbor nodes. Any node who receives this query signal sends back a reply. The nodes involved in these query and reply signals establish and renew their corresponding information in their information tables, including $N$, $L_{i} \mathrm{~s}, A_{i} \mathrm{~s}$ or $M_{C_{-} L i_{-} D K} \mathrm{~s}$, etc.

Set all the measures from me to other nodes via close neighbors to 0 and set all the bandwidth from me to other nodes via close-neighbors to the predetermined minimum values. For those unknown destination nodes to me, reserve adequate memory for the measures and bandwidths.

\section{Routing and Data Transmission Method Based on Percolation}

To provide a reliable route consising of one or multiple paths between any two nodes, link and node outages are very challenging tasks in wireless networks [16][17]. We propose a routing method for the M2M network based on six-degree seperation principle, which is referred to as percolation routing. Suppose that the M2M network has many nodes, and every node has connections with several closeneighbor nodes. The scale of this type of network may change with time, that is, its connection relationship may change with the increasing and disappearing of nodes and link bandwidth may change dynamically with the battery consumption, movement in places of the nodes, and so on.

\subsection{Introduction of Percolation based Routing in M2M network with Small-world Properties}

When a network scale is large and if a node is randomly connected to several nodes in the network, this network can be modeled as a small world network as long as a node is randomly connected to several other nodes. In such a network, when a node wants to send data packets to another node and if the source cannot reach its destination directly, it simply hands the data packets to its close-neighbors which are close to the destination, according to several paths are selected via repeating the close-neighbors' chosen procedure until the probe data packets reach the destination node. In such a way, multiple paths between the source and the destination nodes may be built. Notice that some paths will terminate early before they connect to the destination due to the link outages of network jamming and path halt, part of the probing data packets will disappear in the network without reaching the destination node. On the other hand, to avoid frequent ARQ's feedbacks and improve the flexibility of data transmission, the source data may be encoded using fountain codes [18][19][20]. Specifically, fountain code is a class of rateless codes and the data rate varies according to the instant channel state information contrast to the typical fixed-rate code. With fountain codes, the destination is then capable of successfully decoding the received file if a sufficient number of packets is received, regardless of which packets have been exactly received. This process of routing and data transmission is just like the water seeping in a porous material. As little trickle becomes a stream, and stream becomes torrent, multiple parallel data transmission paths may merge to a high-speed route. Due to this reason, following the literature [21], the proposed routing and data transmission based on sixdegree separation principle are referred to as percolation based routing and data transmission.

\subsection{Percolation Routing Process in M2M network with Small-world Properties}

It is seen that percolation routing is based on probability thus some paths may reach the destination finally whereas other paths will terminate at other nodes, without reaching the destination. Thus the data transmission is inefficient. To avoid the bandwidth loss, we divide the data delivery procedure into two phases: routing phase and data transmission phase. The former phase corresponds to path selection in which probing packets will be transmitted and relayed in the network. In the later phase, paths are available and data transmission will be performed efficiently. To prevent flooding, the number of hops of a path will be restricted up to , for example set this value to six. Moreover, to balance the network resource, we limit the number of maximum paths to , for example set this value to five. $Y X$

Assume that the network initialization is complete, i.e., each node has its initialized routing information table. Then the question of how to discover these paths in an unknown, random wireless network to enable robust multipath routing arises. 


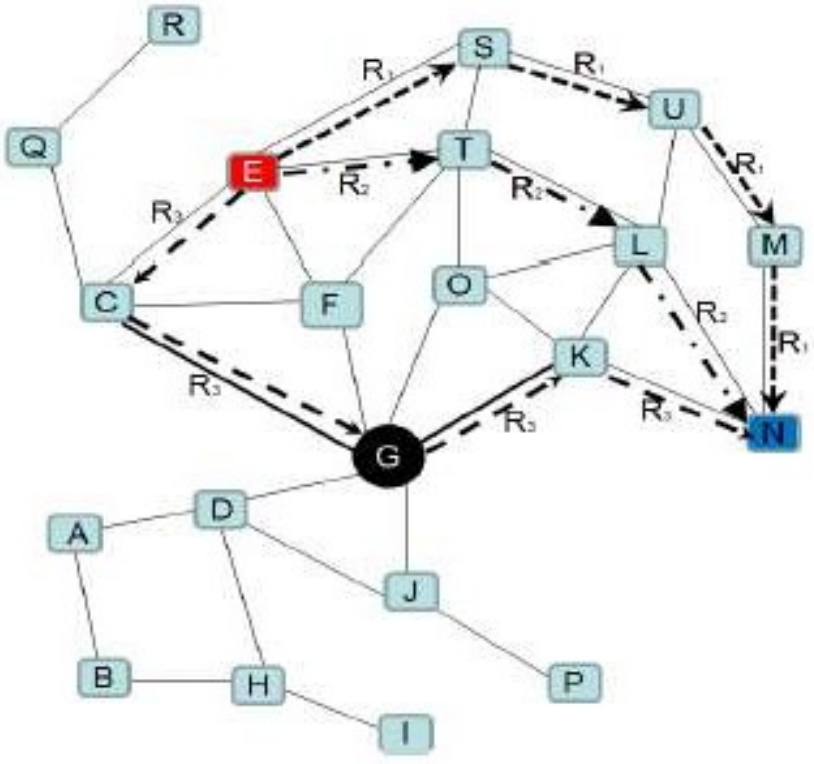

Fig. 2. A routing with multiple paths based on percolation

A stub network structure with its close-neighbor relationships is shown in Fig. 2, which includes A, B, $\mathrm{C}, \ldots \mathrm{U}$, altogether twenty-one nodes. There are several close-neighbors of each node. For example, node $\mathrm{E}$ has four close-neighbors and node $\mathrm{A}$ has two close-neighbors. Node I only has one close-neighbor which means that so long as the node I is not the destination node, the probe data packets which have been received by node I would be dropped by node I. Between any two nodes, one or more paths may be built. For example, though direct connections between Node $\mathrm{E}$ and $\mathrm{N}$ are not available, multiple paths as EF-G-K-N and E-T-O-L-N can be established between these two nodes.

Then the percolation routing procedure is as follows.

Step 1: The source node sends probe packets to its close-neighbor nodes. Probe packet contains the source address, destination address, and a hop counter. Let us reserve positions in the probe for the relay nodes. Set the counter number to zero. $Y$

Step 2: A close-neighbor node receives the probe packet. If the node is not the destination node, it will check the hop counter and switch to the following two cases if the hop counter is less than the preset value $\mathrm{Y}$

Case 1: If there doesn't exist a close-loop closeneighbor node in this selecting path, increase the hop counter by one and the address of the current node is written into the probe packet. Forward the renewed probe packet to a close-neighbor of the maximum measures. Repeat the second step until the destination is reached, then go to the third step.
Case 2: If no any close-neighbor forms a non-closed path, we drop this probe data packet and terminate this routing process.

Step3: The destination node calculates and selects maximum distinct or parallel paths as the routing. The rule to select paths may be based on the least hops, the maximum throughput, and so on. $X$

\section{Conclusions}

We proposed a percolation based routing and data transmission method for the M2M network using the six degrees of separation principle. Under the network structure with "6-degree" distance properties, the proposed scheme consists of two phases: routing phase based on percolation and data transmission phase based on fountain codes. In the routing phase, probes packets are transmitted and flowed in the network, multiple paths are built which form a route. After that, the data file will be fountain encoded and the transmitted data rate is adaptively varying according to the real time channel state information. It is seen that the proposed network routing method either works when a node has the full network architecture or it does when a node contains only local network structure. Since the storage

\section{References}

[1] Stephen M. Dye, "End-to-end M2M", Third Edition, Mind Commerce LLC, Jan 2010, pp. 222224.

[2] I. Cha, Y. Shah, and A. U. Schmidt, "Trust in M2M communication", IEEE Transactions on Vehicle Technology., vol. 4, no. 3, Sept.2009, pp. 69-75.

[3] 3rd Generation Partnership Project (3GPP). Study on facilitation of machine-to-machine communication in 3GPP systems. 3GPP Tech. Rep. 22.868, version 8.0.0, March 2007.

[4] 3rd Generation Partnership Project (3GPP). Feasibility study on remote managements of USIM application on M2M equipment. 3GPP Tech. Rep. 33.812, unpublished draft version 1.4.0, May 2007.

[5] Bob Emmerson, "M2M: The Internet of 50 billion devices", Win-Win Magazine, Jan. 2010, pp.1922.

[6] X. Li, J. Lu, J. Yang and J. An, "A novel routing and data transmission method for stub network of Internet of things based on percolation", , INFOCOM'11, IEEE Conference on Computer 
Communications Workshops on M2MCN, Apr. 2011, pp.201-205.

[7] T. Predojev, J. Alonso-Zarate, M. Dohler, "Energy-delay tradeoff analysis in embedded M2M networks with channel coding", IEEE 21st International Symposium on Personal Indoor and Mobile Radio Communications, Dec. 2010, pp.2733-2738.

[8] M. Martsola, T. Kiravuo and J.K.O. Lindqvist, "Machine to machine communication in cellular networks", IEEE International Conference on Mobile Technology, Applications and Systems, Nov. 2005, pp.1-6.

[9] L. Popova, T. Herpel, W. Koch, "Improving downlink UMTS capacity by exploiting direct M2M data transfer", International Symposium on Modeling and Optimization in Mobile, Ad Hoc and Wireless Networks and Workshops, Apr. 2007, pp.1-8.

[10] C.K. -K Toh, "Ad Hoc mobile wireless networks: protocols and systems", Prentice Hall PTR Upper Saddle River, NJ, USA, pp.324-326. 